Tamas Haidegger, "Taxonomy and Standards in Robotics". In Marcelo H. Ang, Oussama

Khatib, and Bruno Siciliano (eds), Encyclopedia of Robotics, Springer Nature, 2021

\title{
Taxonomy and Standards in Robotics
}

Tamás Haidegger

University Research and Innovation Center (EKIK), Óbuda University

Budapest, Hungary

haidegger@ieee.org

\section{Conflict of Interest statement}

None declared.

\section{Synonyms}

Robot, robotic system, autonomous system, physical agent, Cyber-Physical System, embodied Artificial Intelligence

\section{Definition}

A robot is a complex mechatronic system enabled with electronics, sensors, actuators and software, executing tasks with a certain degree of autonomy. It may be pre-programmed, teleoperated or carrying out computations to make decisions.

\section{Overview and history}

The importance of taxonomy - a systematic classification and naming of a domain becomes obvious when we think about the international cooperation, align of research and market application of the results of a technical field. Robotics has been changing and evolving at rapid pace in the past six decades, yet posing a challenge to the experts to provide an unambiguous definition.

"I can't define a robot, but I know one when I see one."

\section{/Joseph Engelberger, pioneer in industrial robotics/}

The first use of the word robot in the modern sense dates back to 1921, Karel Čapek's Czech playwright RUR (Rossum's Universal Robots) featured cyborgs, human/robot combination that became part of a rebellion that extinguished humanity (Matthews and Greenspan 2020). The term robot or robota had been used in Czech, Hungarian and other regional languages for forced labor. The theme of robot apocalypse has continued to live on ever since in the entertainment industry, and still influences largely the public opinion about robots. The word "robotics" first appeared in Isaac Asimov's 1942 book Runaround, including his famous Three Laws of Robotics, ensuring a safe coexistence of humanoids 
Tamas Haidegger, "Taxonomy and Standards in Robotics". In Marcelo H. Ang, Oussama Khatib, and Bruno Siciliano (eds), Encyclopedia of Robotics, Springer Nature, 20212

and humans. Nevertheless, there is a huge discrepancy in how experts define a robot versus how the public sees it.

\section{Core components of robotics}

Robotics and Artificial Intelligence (AI) are often cited together as the dominating, transformational technology trends of our times. While both terms have been used for several decades, covering partially overlapping domains, the more recent advances in mechatronics, controllers and machine learning jointly opened new, very successful application domains, which require a new taxonomy. A robot traditionally consists of hardware and software components, therefore it has a physical part. The rising importance of machine intelligence and software-based decision making means that AI is gaining more recognition than just being the cognitive controller block of a robot (Haidegger et al. 2019). Moreover, robots many times are just denoted as the embodiment of AI algorithms. Arguably, AI can have numerous application domains even apart from robotics (e.g., Deep Learning in image processing), but herewithin, we focus on the physical application of robotics, with embedded AI supporting it (Karoly et al. 2018).

Robots shall consist of mechanical, electrical and electronic components, constituting of actuators and sensors, assuming that robots are supposed to be physical agents, affecting their environment. The capability to perform an action is considered to be a core component of a robot. An action may be defined as:

one or more operation(s) applied by an agent or team to affect a change in or maintain either an agent's state(s), the environment, or both (IEEE 1872).

A software or a mere virtual simulation can be called a bot or an agent, since it does not take action in the physical environment.

Biological/biochemical structures (nanorobots, microswimmers, etc.) are typically excluded from official definitions.

Robots typically have a well-defined task, e.g., to perform locomotion, manipulation, positioning, reconnaissance or delivery. A task may be defined as:

the specification of a mission, chore, duty, problem, or goal to undertake and accomplish or solve. The task utilizes one or more resources to achieve an intended outcome. The specification may contain constraints on elements from the environment. Tasks are achieved through the execution of one or more actions (IEEE 1872).

Advanced human-machine interaction may also be a robot attribute (although, not exclusive).

Autonomy has been considered a critical component of robotics, yet defining automation is a major challenge in itself (Nof 2009), and resent approaches call towards a domainspecific, gradual quantification on autonomy (called the Level of Autonomy or Degree of Autonomy). The International Organization for Standardization (ISO) even recommends to omit the use of the words "automation" or "automatic" within the frames of their robotic standard, to avoid confusion (Haidegger 2019).

One of the reasons why it is so hard to exactly delimit robotics is the fact that it has changed from being just part of a particular industry domain, and service robotics is now growing at an unprecedented pace, steamed by the rise of consumer robotics (Haidegger et al. 2020). 
Tamas Haidegger, "Taxonomy and Standards in Robotics". In Marcelo H. Ang, Oussama Khatib, and Bruno Siciliano (eds), Encyclopedia of Robotics, Springer Nature, 20213

This means that robotics is joining the megatrends in human history (such as internet, mobile communication, 3D printing), profoundly shaping the entire society, and may help to resolve some of the major challenges we face (Khamis et al 2020).

\section{Definitions and Formalism}

Standardization efforts have been extensive in the robotics domain for the past three decades (Jacobs et al. 2018). It has been a long professional debate to unambiguously define a robot and its components. The traditional ISO 8373 - Robots and robotic devices - Vocabulary standard under ISO first appeared in 1996, only referring to "Manipulating industrial robots", later extended to all robots (in the ISO sense). The responsible Technical Committee (TC) 299 has revised its official definition numerous times in the past years to incorporate all new domains and forms of robots. Their key distinguishing factors are autonomy, mobility and task oriented behavior. The current ISO definition of a robot being "programmed actuated mechanism with a degree of autonomy to perform locomotion, manipulation or positioning”,

wherein autonomy is defined as

"ability to perform intended tasks based on current state and sensing, without human intervention" (ISO 8373).

In the previous versions of the standard more specific requirements were articulated toward the mechatronic structure of the robot (i.e., the Degrees of Freedom to exhibit), and a more generic term "robotic system" was recommended to be used for kind-of-a-robot types not fulfilling the strict ISO definition.

The American National Standards Institute (ANSI) and the Robotic Industries Association (RIA) have their own definition, which is again a heritage from the Industry 3.0 age:

"reprogrammable, multifunctional manipulator designed to move material, parts, tools, or specialized devices through variable programmed motions for the performance of a variety of tasks” (ANSI/RIA R15.06).

To support the relevant R\&D initiatives, the first IEEE standard came out in 2015, the 1872-2015 - IEEE Standard Ontologies for Robotics and Automation, which is now followed by numerous other sub-standards. The IEEE 1872 defines a robot in a broader sense:

"An agentive device (Agent and Device in SUMO) in a broad sense, purposed to act in the physical world in order to accomplish one or more tasks. In some cases, the actions of a robot might be subordinated to actions of other agents (Agent in SUMO), such as software agents (bots) or humans. A robot is composed of suitable mechanical and electronic parts. Robots might form social groups, where they interact to achieve a common goal. A robot (or a group of robots) can form robotic systems together with special environments geared to facilitate their work."

Numerous additional elements and components of a complete robotic system are still under gaining proper definitions, including the tools and concepts of Human-Robot Interfaces (HRI) and interactions. There still may exist a gap (and sometimes overlap) between the definitions, thus IEEE RAS initiated a Standards Strategy meeting series in 2018, under which all the leading Standard Developing Organizations' representative gather to work 
Tamas Haidegger, "Taxonomy and Standards in Robotics". In Marcelo H. Ang, Oussama Khatib, and Bruno Siciliano (eds), Encyclopedia of Robotics, Springer Nature, 20214

on the open issues. The overall aim is to establish a global, harmonized taxonomy standard for the key terms of robotics (Schlenoff 2019).

\section{Robot definitions}

Given the fast pace of technology development, new terms and "buzzwords" emerge, and robotics has become one, resulting in a diversity of definitions (Figure 1). Results have been acquired through a systematic literature research, employing the keywords "robot + definition", "robotics + definition", "robotic system + definition" on Google Scholar, IEEE Xplore, Springer Link, PubMed and Google search. It was extended with an online standards database search, rostered, filtered and manually evaluated. Notable (i.e., scientifically and technically sound and coherent) definitions were collected for the term robot to be put into the bigger context of robotic $\mathrm{R} \& \mathrm{D}$ (Table 1).

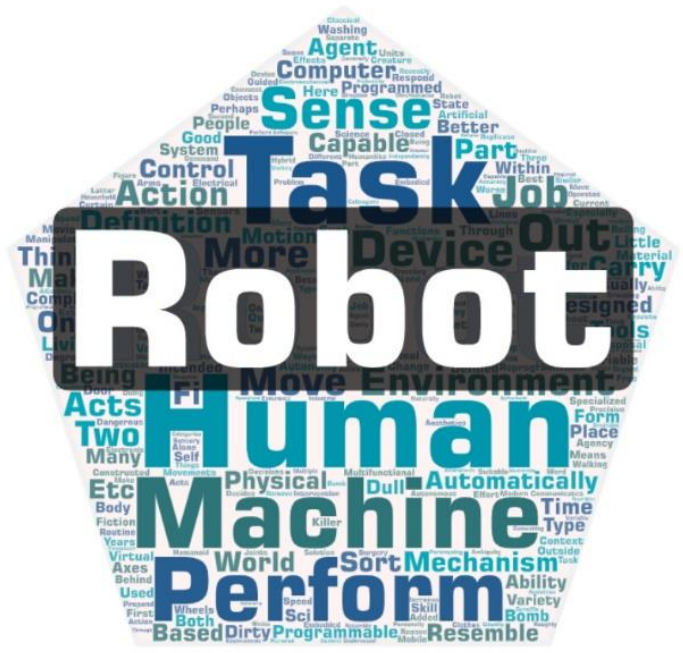

Figure 1. Wordle forged based on the identified top 30 robot definitions.

Official robot definitions remain very conservative, as the aim of standards is generally to codify an already widely accepted consensus.

Table 1. Most relevant robot definitions identified based on a systematic literature research. Sources are indicated, where available. E: expert type definition, L: layman type definition.

\begin{tabular}{|c|c|c|c|c|c|}
\hline No & Tag (short name) & Year & Type & Definition & Source \\
\hline 1 & $\begin{array}{l}\text { ISO 8373:2012 } \\
\text { FDIS }\end{array}$ & 2012 & $E$ & $\begin{array}{l}\text { A robot is an actuated } \\
\text { mechanism } \\
\text { programmable in two or } \\
\text { more axes with a degree } \\
\text { of autonomy, moving } \\
\text { within its environment, to } \\
\text { perform intended tasks }\end{array}$ & https://www.iso.org/standard/55890.html \\
\hline 2 & $\begin{array}{l}\text { ISO } 8373: 2020 \\
\text { FDIS }\end{array}$ & 2020 & $E$ & $\begin{array}{l}\text { Programmed actuated } \\
\text { mechanism with a degree } \\
\text { of autonomy to perform }\end{array}$ & https://www.iso.org/standard/55890.html \\
\hline
\end{tabular}


Tamas Haidegger, "Taxonomy and Standards in Robotics". In Marcelo H. Ang, Oussama Khatib, and Bruno Siciliano (eds), Encyclopedia of Robotics, Springer Nature, 20215

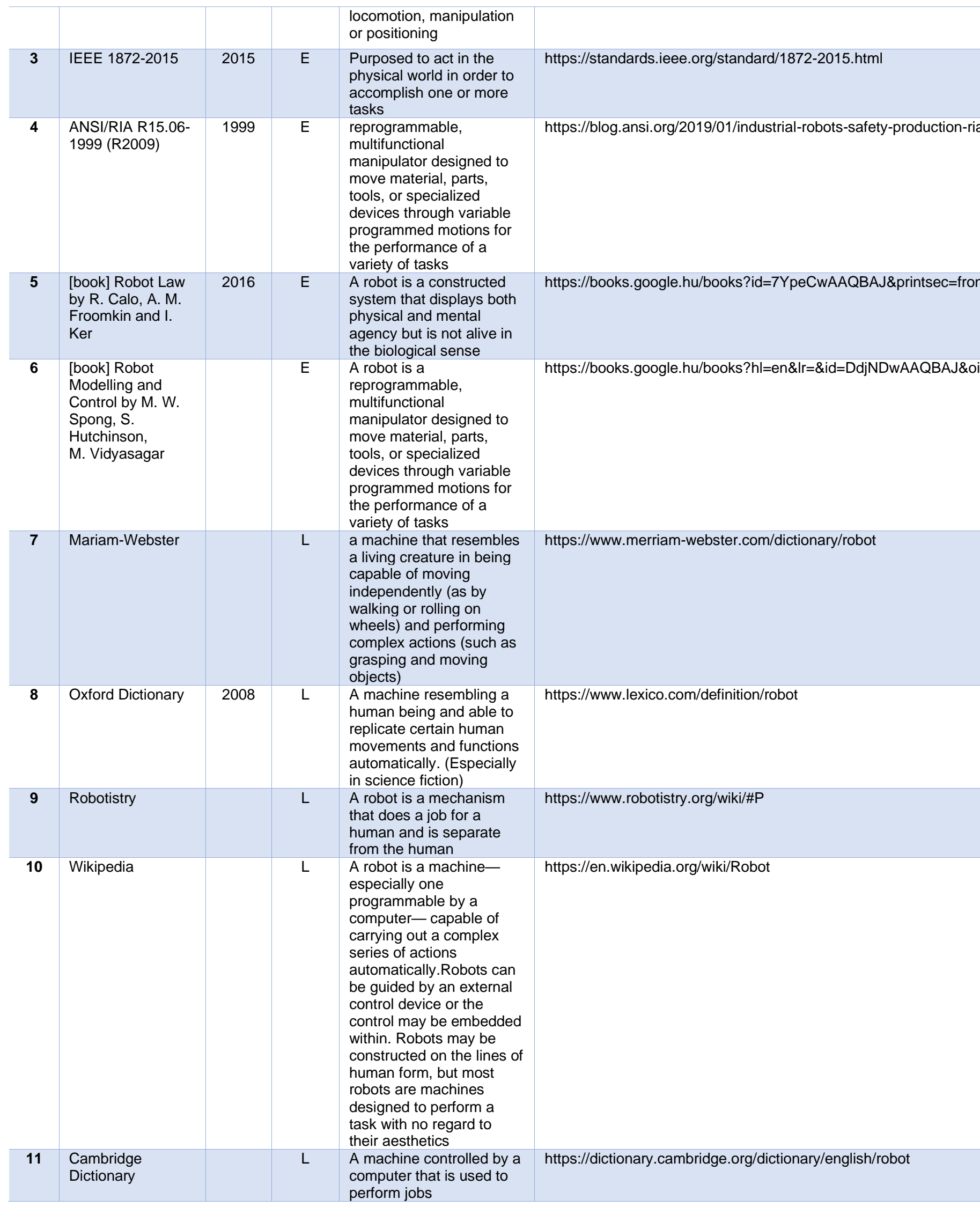


Tamas Haidegger, "Taxonomy and Standards in Robotics". In Marcelo H. Ang, Oussama Khatib, and Bruno Siciliano (eds), Encyclopedia of Robotics, Springer Nature, 20216

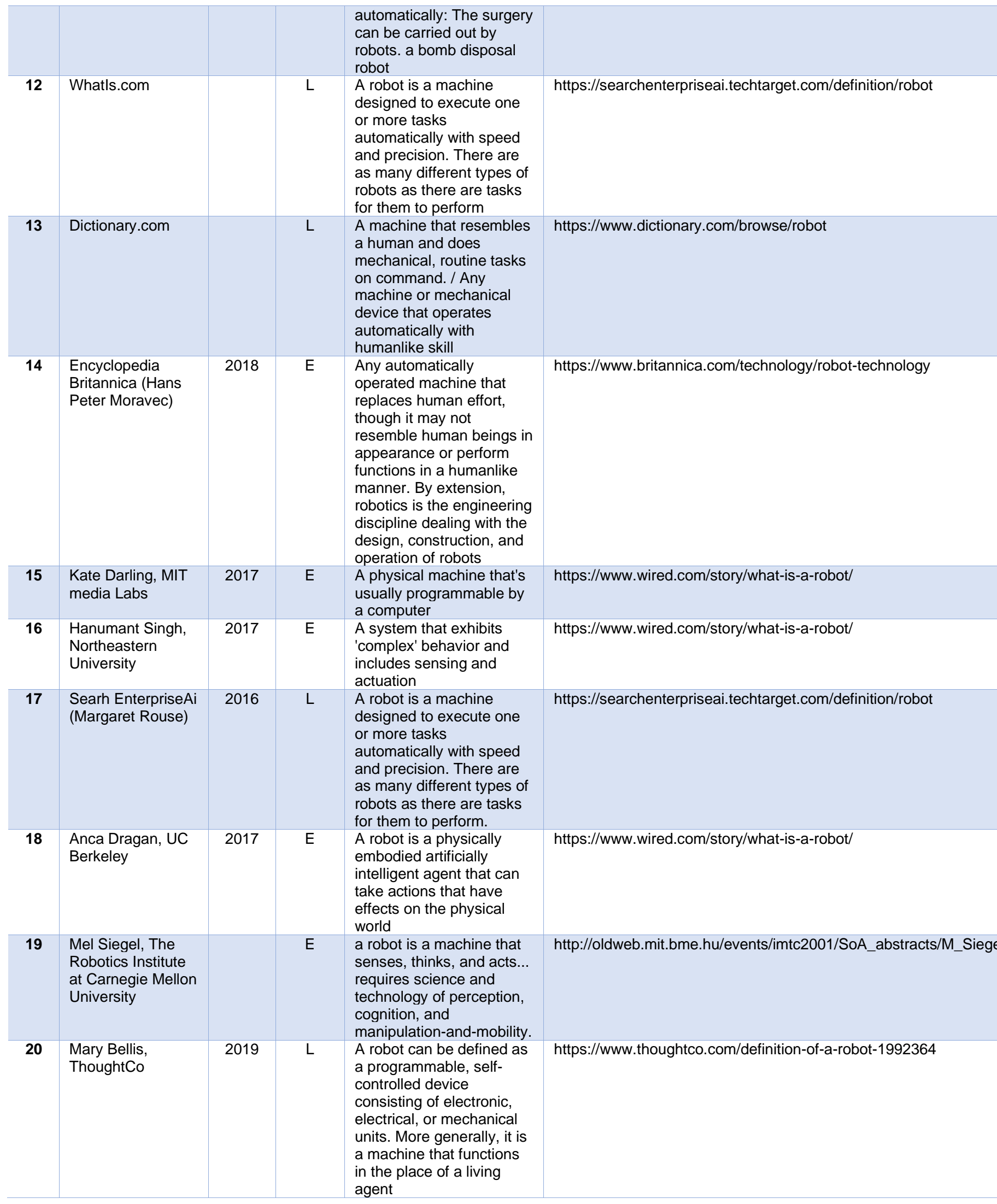


Tamas Haidegger, "Taxonomy and Standards in Robotics". In Marcelo H. Ang, Oussama Khatib, and Bruno Siciliano (eds), Encyclopedia of Robotics, Springer Nature, 20217

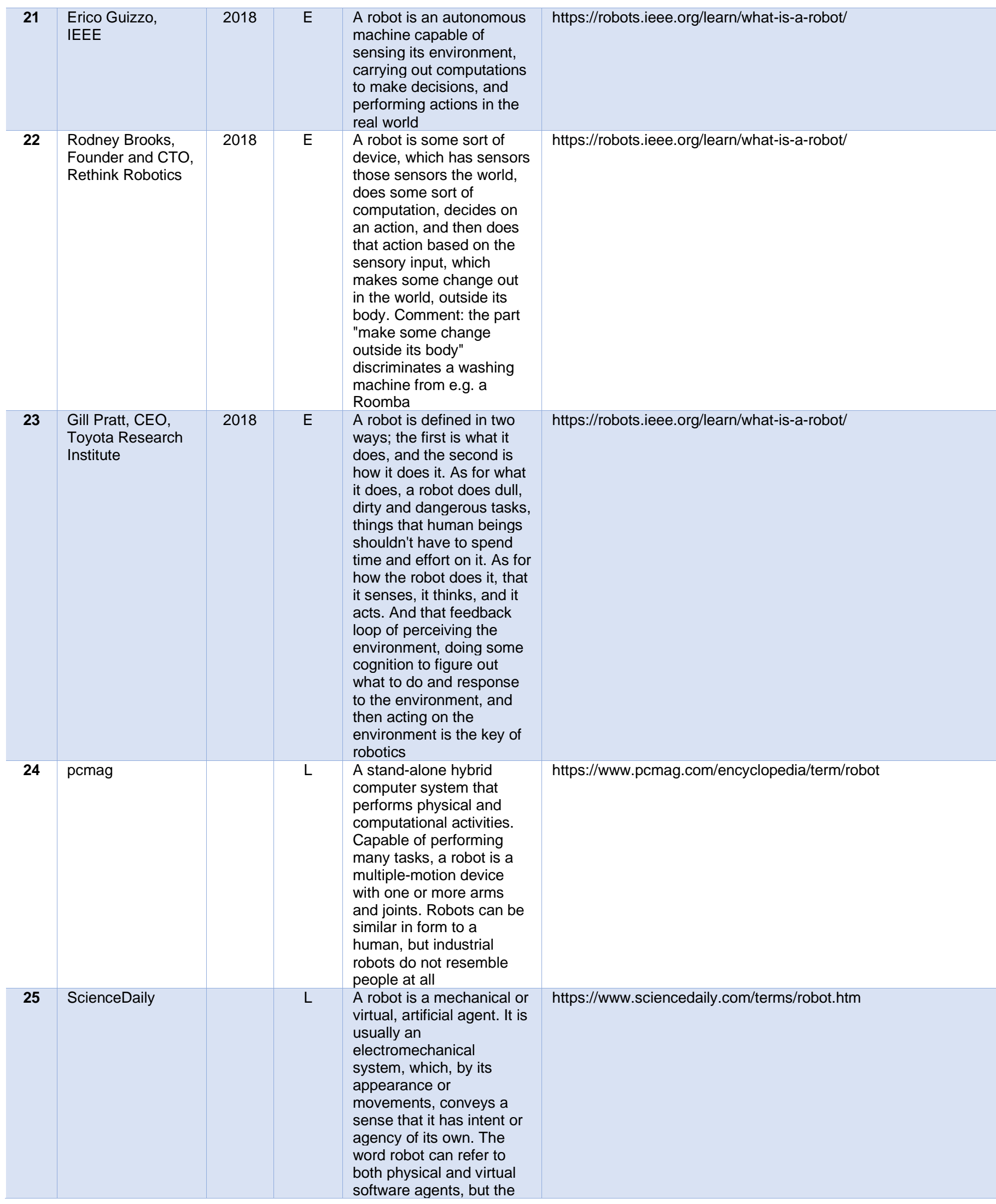


Tamas Haidegger, "Taxonomy and Standards in Robotics". In Marcelo H. Ang, Oussama Khatib, and Bruno Siciliano (eds), Encyclopedia of Robotics, Springer Nature, 20218

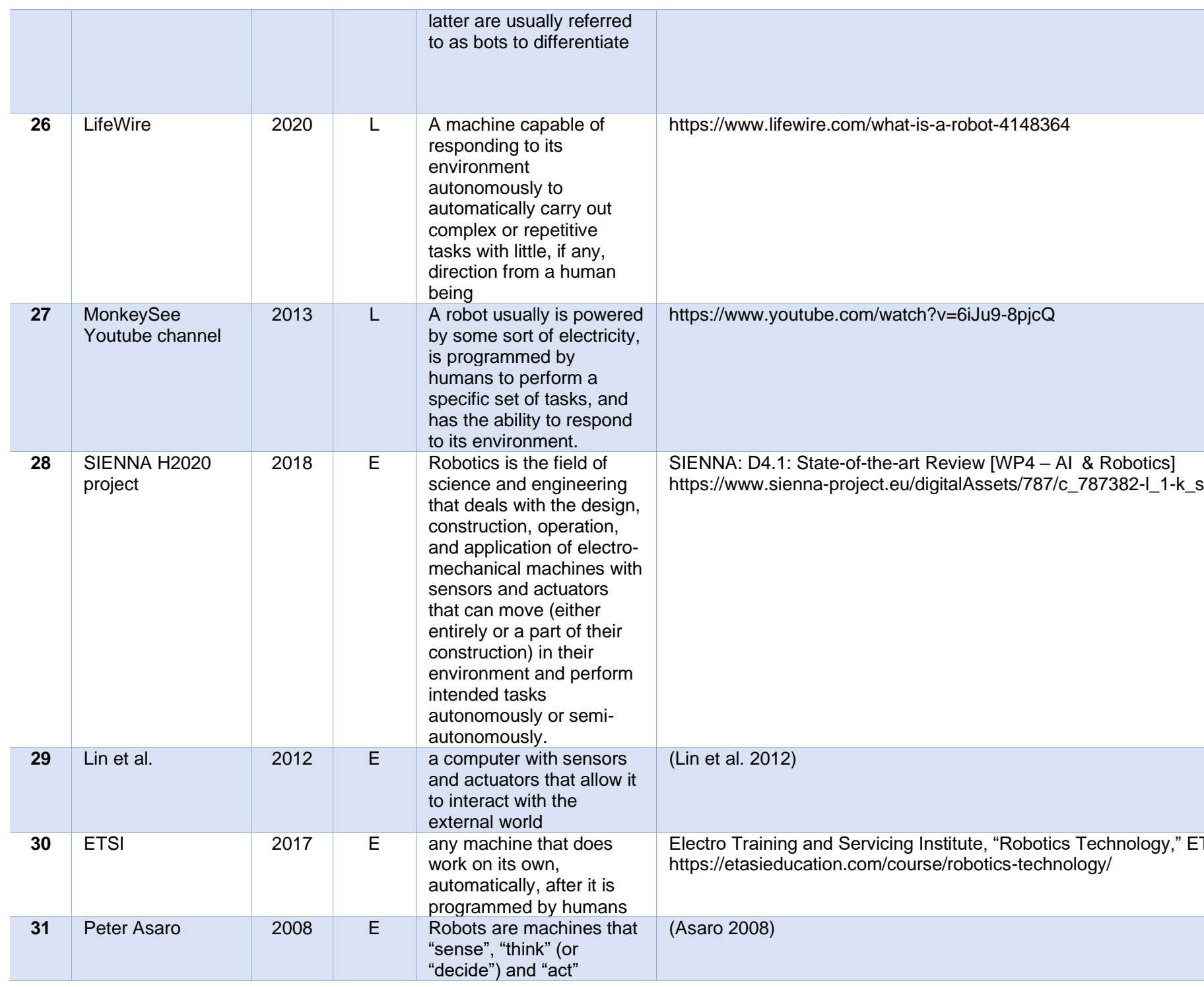

\section{Sub-domains of robotics, ambiguities and border line issues}

The ISO 8373, as the most widely used reference, also distinguishes core application areas of robotics, such as industrial and service domains, while excluding household appliances, military systems, toys and simple machines. Nevertheless, these still generate a lot of public attention, and sometimes, the borderline is very fine.

According to the standard (ISO 8373), an industrial robot is being defined as

"Automatically controlled, reprogrammable multipurpose manipulator(s), programmable in three or more axes, which can be either fixed in place or mobile for use in industrial automation applications",

Other definitions focus on the particular applications: "Industrial robotics deals with the application of robots and manipulators for welding, casting, painting, surface protection, machining, assembly, and many other industrial processes which are physically demanding, hazardous, or monotonous." (Morecki \&Knapczyk, 1999) 
Tamas Haidegger, "Taxonomy and Standards in Robotics". In Marcelo H. Ang, Oussama Khatib, and Bruno Siciliano (eds), Encyclopedia of Robotics, Springer Nature, 20219

A service robot being (ISO 8373):

"Robot that performs useful tasks for humans or equipment excluding industrial automation applications"

A military robot definition is called (Ashokkumar, M., \& Thirumurugan 2018)

"Autonomous robots or remote-controlled mobile robots designed for military applications, from transport to search \& rescue and attack."

Certain application domains have already started to develop standard definition, notably e.g., for medical robots. In 2018, the IEEE Engineering in Medicine and Biology (EMB) (co-sponsored by the IEEE RAS) started a new standards working group: IEEE P2730 Standard for Classification, Terminologies, and Definitions of Medical Robots, with the scope to specify the category, naming and definition of medical robots (Haidegger 2019).

According to ISO, a medical robot is

"A robot with medical intended use".

It is very critical to see that this categorization may be overruled by national and regional laws and regulations. In Europe, while for most robot applications, the Machinery Directive (DIRECTIVE 2006/42/EC) is the appropriate legal guiding document, all medical robots are regulated as diagnostic and treatment technology, therefore the Medical Device Regulation (REGULATION (EU) 2017/745) is compulsory to be followed for safety and reliability compliance. Arguably, it is very hard in many cases to distinguish between medical and non-medical use of the same technology (Virk \& Haidegger 2012).

In other application domains, historical heritage determines public opinion. For example, while a cargo airplane in autopilot mode fulfil all possible requirements towards a drone or more generically speaking, an Unmanned Aerial Vehicle (UAV), which is a robot, people many times refuse that term. Similarly, people refuse to call self-driving cars robots, while they fulfil all requirements of a mobile robot at higher Levels of Autonomy (Drexler et al. 2019).

\section{Safety, performance and ethical standards for robots}

While taxonomy standards are limited for robots (and even ISO considers abandoning it), there are numerous industry standards essential for the safe and efficacious application of robot technologies. For the traditional industrial manipulators, probably the most important safety standards came from the current ISO TC 299, the ISO 10218-1 - Robots and robotic devices - Safety requirements, which is regularly reviewed by the experts, while the currently rising domain of collaborative robotics is still just addressed by a technical report (ISO TR 15066:2015 - Robots and robotic devices - Safety requirements for industrial robots - Collaborative operation). The full set of essential performance and basic safety standards released for both the industrial, service and medical domains (ISO 18646, ISO 13482, etc), is now being amended by new modularity standards, e.g., the ISO/FDIS 22166-1 - Robotics - Part 1: Modularity for service robots (Jacobs et al. 2018).

The numerous Standard Development Organizations, such as ANSI/ITSDF, ASTM, ANSI/RIA, IEEE, ISO and SEA have released over 140 robotics related standards altogether (as tracked by the Robotistry http://robotistry.org/standards/StandardsList.html). 
Tamas Haidegger, "Taxonomy and Standards in Robotics". In Marcelo H. Ang, Oussama Khatib, and Bruno Siciliano (eds), Encyclopedia of Robotics, Springer Nature, 202110

Concerning the non-technical standards, remarkable initiatives have been launched to address the ethical issues of robotics and automation. The IEEE is drafting relevant standards within the frames of the IEEE Global Initiative on Ethics of Autonomous and Intelligent Systems (https://standards.ieee.org/industry-connections/ec/autonomoussystems.html), building up the principles of Ethically Aligned Design (IEEE SA 2016). This has resulted in the three pillars of the EAD defined as:

- Universal human values;

- Political self-determination data agency;

- Technical dependability.

This initiative also resulted the standard family P7000 (https://ethicsinaction.ieee.org/p7000/), primarily within the IEEE P7000 - Model Process for Addressing Ethical Concerns During System Design, IEEE P7001 - Transparency of Autonomous Systems and the particularly robot focused IEEE P7007 - Ontological Standard for Ethically Driven Robotics and Automation Systems and the IEEE P7008 Standard for Ethically Driven Nudging for Robotic, Intelligent and Autonomous Systems. Other major AI and robot ethics initiatives include the Trusting AI platform from IBM (https://www.research.ibm.com/artificial-intelligence/trusted-ai/), the Campaign To Stop Killer Robots (https://www.stopkillerrobots.org/) and the Stanford Encyclopedia of Philosophy: Ethics of Artificial Intelligence and Robotics (https://plato.stanford.edu/entries/ethics-ai/).

\section{Discussion and future directions}

Arguably, it is hard to define exactly the term robot, given their various structures, functions, applications and appearances. In the advent of a new robotics era marked by the generation of "robotic natives", there are several contributing factors that should be observed (Boesl \& Liepert 2017). The constellation of a set of powerful technologies will lead to the appearance of new generations of robots, having more services and more overlap with Industry 4.0, big data, rapid prototyping, AR/VR, IoT, simulation, cloud computing and other domains. Moving beyond the traditional ISO definitions, walls between the narrow mechatronics meaning of robotics and the neighboring ICT technologies is completely disappearing. Latest robot generations either in industrial or service domain are depending on computing and telecommunications solutions that are extensively used in general purpose IT. The - conventionally very conservative-robotics community became open to exploit the synergies of fields like computer science, AI and the cognitive mechatronics.

Besides the pure technological aspects, we also witness a paradigm shift in the human compatibility of robotics, robot builders are optimizing the cognitive couplings between humans and robots beyond the traditional HRI. As a result, robots evolve from complete isolation to interconnected, human-centric Cyber-Physical Systems (CPS), marked by the advancement of radical new technology breakthroughs presented and reviewed hereafter.

\section{Acknowledgments}

The author thanks Tamas D. Nagy for his help in the data collection. 
Tamas Haidegger, "Taxonomy and Standards in Robotics". In Marcelo H. Ang, Oussama

Khatib, and Bruno Siciliano (eds), Encyclopedia of Robotics, Springer Nature, 202111

\section{References}

Asaro, Peter. "How just could a robot war be." Current issues in computing and philosophy (2008): 50-64.

Ashokkumar, M., \& Thirumurugan, T. (2018). Integrated IoT based design and android operated multi-purpose field surveillance robot for military use. In International Conference for Phoenixes on Emerging Current Trends in Engineering and Management (PECTEAM 2018). Atlantis Press.

D. B. O. Boesl and B. Liepert, "4 Robotic Revolutions - proposing a holistic phase model describing future disruptions in the evolution of robotics and automation and the rise of a new Generation 'R' of Robotic Natives," 2016 IEEE/RSJ International Conference on Intelligent Robots and Systems (IROS), Daejeon, 2016, pp. 1262-1267, doi: 10.1109/IROS.2016.77592097

Drexler, D. A., Takács, A., Nagy, T. D., \& Haidegger, T. (2019). Handover Process of Autonomous Vehicles-technology and application challenges. Acta Polytechnica Hungarica, 16(9), 235-255.

Haidegger, T. (2019). Autonomy for surgical robots: Concepts and paradigms. IEEE Transactions on Medical Robotics and Bionics, 1(2), 65-76.

Haidegger, T., Galambos, P., \& Rudas, I. J. (2019). Robotics 4.0-Are we there yet? In 2019 IEEE 23rd International Conference on Intelligent Engineering Systems (INES) (pp. 117-124). IEEE.

Haidegger, T., Virk, G. S., Herman, C., Bostelman, R., Galambos, P., Györök, G., \& Rudas, I. J. (2020). Industrial and medical cyber-physical systems: Tackling user requirements and challenges in robotics. In Recent Advances in Intelligent Engineering (pp. 253-277). Springer, Cham.

IEEE 1872 Committee RAS/SC - Standing Committee for Standards (2015) IEEE 1872-2015 - IEEE Standard Ontologies for Robotics and Automation, IEEE

IEEE SA Global Initiative (2016). Ethically Aligned Design. $1^{\text {st }}$ Edition (EAD1e), 2016, pp. 1-138. IEEE.

ISO FDIS 8373:2020 - Robots and robotic devices - Vocabulary. ICS: 01.040.25 Manufacturing engineering (Vocabularies). 25.040.30 Industrial robots. Manipulators

Jacobs, T., Veneman, J., Virk, G. S., \& Haidegger, T. (2018). The flourishing landscape of robot standardization. IEEE Robotics \& Automation Magazine, 25(1), 8-15.

Károly, A. I., Fullér, R., \& Galambos, P. (2018). Unsupervised clustering for deep learning: A tutorial survey. Acta Polytechnica Hungarica, 15(8), 29-53. 
Tamas Haidegger, "Taxonomy and Standards in Robotics". In Marcelo H. Ang, Oussama Khatib, and Bruno Siciliano (eds), Encyclopedia of Robotics, Springer Nature, 202112

Khamis A., Meng J., Wang J, Azar A.T., Prestes E., Li H., Hameed I. and Haidegger T. (2020, in press). AI and Robotics in the Fight Against COVID-19 Pandemic. Chapter in Modelling, Control and Drug Development for COVID-19 Outbreak Prevention, book edited by Ahmed Taher. Studies in Systems, Decision and Control by Springer.

Lin, Patrick, Keith Abney, and George A. Bekey. "Current trends in robotics: Technology and Ethics." (2012): 17-34.

Matthews P., Greenspan S. (2020) Robots in Society. In: Automation and Collaborative Robotics. Apress, Berkeley, CA

Morecki A., Knapczyk J. (1999) Industrial Robotics. In: Morecki A., Knapczyk J. (eds) Basics of Robotics. International Centre for Mechanical Sciences (Courses and Lectures), vol 402. Springer, Vienna. https://doi.org/10.1007/978-3-7091-2532-8_14

Nof, S. Y. (2009). Automation: What it means to us around the world. In Springer handbook of automation (pp. 13-52). Springer, Berlin, Heidelberg.

Schlenoff, C. (2019) Results of the IEEE RAS Standards Strategy Meeting [Standards], in IEEE Robotics \& Automation Magazine, vol. 26, no. 1, pp. 105-107, March 2019, doi: 10.1109/MRA.2019.2891184.

Virk, G. S., \& Haidegger, T. (2012). Classification guidelines for personal care robotsmedical and non-medical applications. In Proc. of the 2012 IEEE IROS Workshop on Safety in Human-Robot Coexistence \& Interaction (pp. 33-36). 